\title{
The influence of starspots activity on the determination of planetary transit parameters
}

\author{
Adriana Silva-Válio ${ }^{1}$ \\ ${ }^{1}$ CRAAM, Mackenzie University, São Paulo, Brazil \\ email: avalio@craam.mackenzie.br
}

\begin{abstract}
As a planet eclipses its parent star, dark spots on the surface of the star may be occulted, causing a detectable variation in the transit light curve. There are basically two effects caused by the presence of spots on the surface of the star which can alter the shape of the light curve during transits and thus preclude the correct determination of the planet physical and orbital parameters. The first one is that the presence of many spots within the latitude band occulted by the planet will cause the depth of the transit in the light curve to be shallower. This will erroneously result in a smaller radius for the planet. The other effect is that generated by spots located close to the limb of the star. In this case, the spots will interfere in the light curve during the times of ingress or egress of the planet, causing a decrease in the transit duration. This in turn will provide a larger value for the semi-major axis of the planetary orbit. Qualitative estimates of both effects are discussed and an example provided for a very active star, such as CoRoTo-2.
\end{abstract}

Keywords. stars: activity, stars: spots, planetary systems

\section{The spotted star model}

During one of its transits, an extrasolar planet may pass in front of a stellar group of spots. Spots are regions of high concentration of magnetic fields, being cooler, and therefore darker, than the surrounding photosphere. Because a darker region of the stellar surface is being occulted when the planet crosses in front of the spot, a small bump may be observed in the bottom of the transit light curve.

I have used the model described in Silva (2003), that simulates the star as a 2-D image with quadratic limb darkening. The planet is assumed to be a dark disc of radius $R_{p}$, with no emission. Finally, the spots are modeled by three parameters: (i) Intensity, as a function of stellar intensity at disk center $\left(I_{c}\right)$; (ii) Size, as a function of planet radius; and (iii) Position: Latitude (restricted to the transit path) and Longitude.

The transit light curve is calculated every two minutes, when the planet is centered at a given position in its orbit. The orbit, considered to be circular, is described by the semi-major axis (or orbital radius) and inclination angle. The integrated flux is calculated by summing the intensity in all the pixels, thus yielding the light curve. The three panels of Figure 1 show the effect of varying one of the model parameters:

- Planet radius: The observed transit depth is $\Delta I / I=\left(R_{p} / R_{s}\right)^{2}$, where $R_{s}$ is the star radius. Planets with larger radius will produce deeper transits. Jupiter size planets produce a $1 \%$ decrease in the light curve intensity of a solar like star.

- Orbital radius: The orbit is assumed to be circular, that is zero eccentricity. The larger the orbital radius, the smaller the phase interval of the transit. This is equivalent to saying that larger orbital distances result in shorter transits (in time) for the same orbital period. 

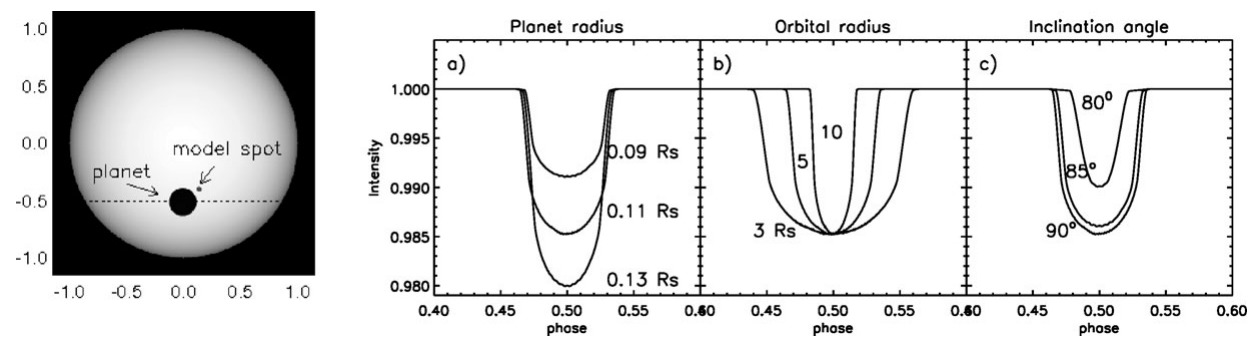

Figure 1. Left: An example of the simulate star with a small spot and the planet. The effect of varying the planet parameters: (a) planet radius; (b) semi-major axis; and (c) inclination angle.

- Inclination angle: For a transit to be detect, the orbital inclination angle has to be very close to $90^{\circ}$, otherwise no transit will be observed. The smaller the angle, the shorter the phase interval of the transit. Triangular shaped transits result from grazing eclipses, that is, inclination angles less than about $80^{\circ}$.

\section{Transit depth and width}

As mentioned above, the transit depth depends on the ratio of the planet to the star radii. As an illustration, the result from the simulation of a star with 9 spots of constant radius equal to $0.5 R_{p}$ and intensity of half the star central maximum intensity is shown as a dotted line in the left panel of Figure 2. A heavily spotted star will present a shallower transit. Therefore the fit to this light curve results in a smaller radius for the planet (gray solid line). In the present simulation, the estimated planet radius from the light curve changed from 0.1 to $0.093 R_{s}$, a $7 \%$ decrease in the radius determination.
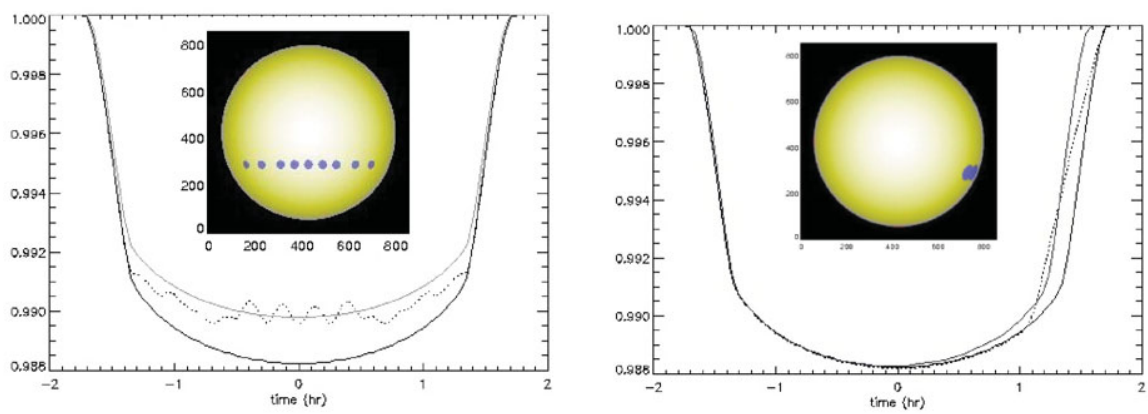

Figure 2. Left: Model light curves representing the simulated model of a spotless star (black line), and that of a star with nine spots (shown in the inset): data (dots) and fit (gray line). Right: Model light curves representing the simulated model of a spotless star (black line), and that of a star with two limb spots (shown in the inset): data (dots) and fit (gray line).

The next simulation includes two spots close to the limb of the star, as shown in the right panel of Figure 2. In this case, the width of the transit, or its duration will seem smaller (dotted line). The gray solid line in the figure represents a misleading fit to the transit light curve. Thus, when the transit fitting is performed the resulting semi-major axis of the orbit will be larger. In this simulation the inferred orbital radius increased from 7 to $7.3 R_{s}$, a $4 \%$ increase in the orbital radius.

\section{Application to CoRoT-2}

CoRoT-2 is known to be a very young star and due to its intense spot activity, many spot on its surface are present at any given time. This star has an orbiting planet such 
that 77 transits of the planet were observed with 32 second resolution by the CoRoT $\dagger$ satellite (Baglin et al. 2006). According to Alonso et al. (2008) the planet has a radius of $1.47 R_{J u p}$ and orbits the star every 1.743 days on a circular orbit (Bouchy et al. 2008) of radius $6.7 R_{s}$ with an inclination angle of $87.84^{\circ}$. This inclination angle implies that the path of the transit overlays the star at a latitude of $14.6^{\circ}$.

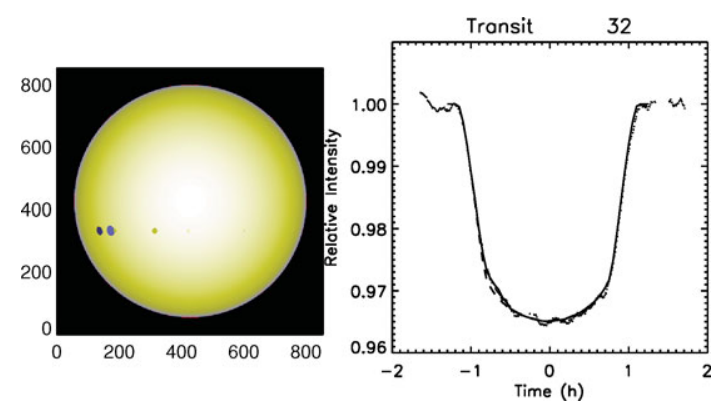

Figure 3. Left: Model of the star with a few spots. Right: The 32nd transit light curve for CoRoT-2 (dots) and the model for a spotless star (dashed line) and the fit to the data (solid line) for the model shown in the left panel.

To estimate the best radius for the planet, the deepest transit was sought. Of all the 77 transits detected, the 32nd transit displays the lower light variation (Figure 3 ). This transit is believed to have the least number of spots on its surface within the transit latitudes during the whole period of observation. The dashed curve on the figure represents the model without any spot whereas the solid line refers to the fit of the light curve with basically three small spots. To obtain this fit light curve it was necessary to use a planet radius of 0.172 stellar radius, instead of the 0.1667 stellar radius (Alonso et al. 2008). This represents an increase of about $3 \%$ in the planet radius, and is equivalent to 1.52 $R_{\text {Jup }}$.

\section{Conclusions}

Spots on the stellar surface are responsible for basically two effects that can alter the shape of the light curve during transits: (1) smaller depth and (2) shorter duration. These effects can preclude the correct determination of the planet physical and orbital parameters. The presence of many spots within the latitude band occulted by the planet will result in a light curve with shallower transit depth, thus erroneously yielding a smaller radius for the planet. The other effect is that caused by spots located close to the limb of the star that interferes in the light curve at times of ingress or egress of the planet, causing a decrease in the transit duration. This in turn will provide a larger value for the planetary orbital radius. In the simulations presented here, these effects are of the order of a few percent $(3-7 \%)$ in both cases.

\section{References}

Alonso, R., Auvergne, A., Baglin, A. et al. 2008, A\&SA, 482, L21

Baglin, A. et al. 2006, 36th COSPAR Scientific Assembly, 36, 3749

Bouchy, F., Queloz, D., Deleuil, M., Loeillet, B., Hatzes, A. P. et al. 2008, A\&̈A, 482, L25

Silva, A. V. R. 2003, ApJ, 585, L147

$\dagger$ CoRoT is a space project operated by the French Space Agency, CNES, with participation of the Science Programme of ESA, ESTEC/RSSD, Austria, Belgium, Brazil, Germany, and Spain. 